\title{
Expression of a Rap2.12 like-1 ERF gene during adventitious rooting of chestnut and oak microshoots
}

\author{
Silvia Valladares ${ }^{\mathrm{a}, *}$, Elena Varas ${ }^{\mathrm{a}, *}$, Jesús M. Vielba ${ }^{\mathrm{b}}$, Nieves \\ Vidal $^{\mathrm{b}}$, Veronica Codesido ${ }^{\mathrm{c}}$, Ricardo Castro ${ }^{\mathrm{b}}$ and Conchi Sanchez ${ }^{\mathrm{d}}$
}

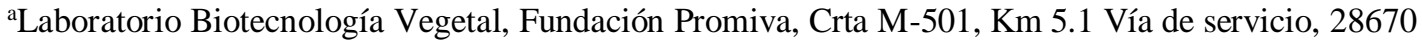
Villaviciosa de Odón, Spain;

bepartment of Plant Physiology, Instituto de Investigaciones Agrobiológicas de Galicia (IIAG-CSIC), Apartado 11, 15780, Santiago de Compostela, Spain;

cDepartment of Breeding and Cultivation, Phytoplant Research S.L., Calle Astrónoma Cecilia Payne, Edificio Centauro, Módulo B-1, Rabanales 21, 14014 Córdoba, Spain;

${ }^{\mathrm{d} D e p a r t m e n t}$ of Plant Physiology, Instituto de Investigaciones Agrobiológicas de Galicia, Avda de Vigo s/n, Santiago de Compostela, A Coruña, 15705, Spain. Email: conchi@iiag.csic.es

* Silvia Valladares and Elena Varas contributed equally to this work.

Israel Journal of Plant Sciences

https://dx.doi.org/10.1163/22238980-20191119

\begin{abstract}
Adventitious rooting of cuttings is a complex developmental process in forest species, with several exogenous and endogenous factors influencing the outcome of the process. In this study we applied an in vitro working system, comprising two lines of microshoots with the same genotype but at a different ontogenetic stages, in two different tree species (chestnut and oak). We analyzed the expression of a gene encoding an AP2/ERF transcription factor from group VII in the initial hours of the adventitious rooting induction, both in rooting competent and incompetent microshoots. The analysis revealed that expression of this gene is related to wounding, ontogenetic stage and auxin in a complex and species-specific manner. Putative induction of the gene by auxin was also analyzed in the presence of naphthyl-phthalamic acid (NPA), an auxin transport inhibitor. In situ expression analysis in chestnut relates the gene activity to cambial divisions and root primordia in rooting competent microshoots, as well as in the root apex. The putative role of the gene during adventitious roots formation is discussed.
\end{abstract}

Keywords: Adventitious roots; AP2/ ERF gene; auxin; gene expression; naphthyl-phthalamic acid; root primordia

\section{Introduction}

The induction of adventitious rooting (AR) in cuttings is an essential step in the successful clonal propagation of selected genotypes and is of great economic importance in the forestry and horticultural industries. This developmental process is affected by endogenous factors, including (but not limited to) the physiological status of the plant, the phytohormone content and distribution, the wounding response, aging and the presence of e.g. reactive oxygen and nitrogen species (ROS and RNS), phenolic compounds and polyamines. AR is also influenced by exogenous factors, such as light conditions, temperature, mineral nutrition and certain biotic interactions with bacteria or fungi (Geiss et al. 2009; Guan et al. 2015). Moreover, the different hormones establish a complex crosstalk at each step of the process (Lakehal and Bellini 2019). Among these factors, auxin emerges as a key player in the formation of adventitious roots (ARs), and its dominant role in this organogenic process has been clearly established (reviewed in Druege et al. 2016). Nonetheless, auxins induce several responses that involve changes in the expression of hundreds of genes in different cell types, not only in those involved in the AR process. In addition, the preponderant effect of auxins can mask the importance of other factors 
that may also significantly influence the formation of new roots. In order to clarify these responses, the use of Polar Auxin Transport (PAT) inhibitors may be helpful to highlight the effect of other factors involved in the AR process. PAT inhibitors hinder the basipetal transport of auxin, perturbing its distribution and therefore altering the ongoing developmental programs (Saniewski et al. 2014). NPA is the most common PAT inhibitor used in auxin research, although its specific mode of action has not yet been clearly defined (Teale and Palme 2018).

In woody species, another relevant factor that must also be considered is the effect of maturation or phase change on the regeneration competence (Hackett and Murray 1996). Basal shoots taken from the base of the trunk generally exhibit juvenile-like characteristics and root well in response to exogenous auxin. By contrast, ontogenetically mature cuttings from the crown branches scarcely form ARs when subjected to the same treatments, particularly in recalcitrant species. Recalcitrance, the inability to respond to a stimulus applied, constitutes a major limitation for the clonal propagation of many woody species (Bonga 1987; Díaz-Sala 2014). The different rooting responses of juvenile-like and mature tissues have been identified in the two species under study here, i.e. chestnut (Castanea sativa Mill.) and oak (Quercus robur L.) (Sánchez and Vieitez 1991; Ballester et al. 1999, 2009; Vidal et al. 2003). Auxin induces the dedifferentiation of specific cells in both juvenile-like and mature microshoots, particularly in the cambium region and in cambial derivatives that eventually divide to give rise to ARs in juvenile shoots or calli in mature shoots. (Ballester et al. 1999; Vidal et al. 2003).

All of the aforementioned factors provide stimuli to the plant, but the actual extent to which each influences the process has not been clearly defined. Moreover, the signalling pathways involved in the response to these factors may be closely intertwined, adding a significant level of complexity to the characterization of the effect of any particular factor. In order to integrate the information provided by these physiological and environmental cues, expression of transcription factors (TFs) is of paramount importance. The information that tissues receive from these inner and outer signals is translated into specific gene expression programs. The TFs involved in those programs are key elements in the subsequent responses, as they regulate the expression of many downstream genes, thereby determining the activation of the proper developmental programs. Hence, the identification and analysis of TFs involved in AR is crucial for a better understanding of this developmental program.

To date, only a few TFs have been characterized in relation to their role during AR in cuttings of woody species, particularly during the initial events that lead to dedifferentiation of specific cells within or close to the cambial zone (Legué et al. 2014). These cells will change their fate when correctly stimulated and will become the founder cells of the developing new ARs (DíazSala 2014). One of the early efforts in this field led to the identification of two Scarecrow-like genes belonging to the GRAS family of TFs. These genes (CSSCL-1 from C. sativa and PrSCL1 from Pinus radiata) were induced within the first $24 \mathrm{~h}$ of AR in response to auxin treatment, suggesting a possible conserved role for SCLI in the initial events of the AR process in these distantly-related species (Sánchez et al. 2007). Moreover, transcripts of both genes were preferentially localized in the cambial region of rooting-competent shoots, reinforcing their putative role in the early events leading to the reprogramming of specific cells surrounding the cambial zone that will eventually become the founder cells of the ARs (Solé et al. 2008; Vielba et al. 2011).

Most of the available information about the involvement of AP2/ERF TF in controlling adventitious root formation is derived from work with the model tree Populus trichocarpa, because the full characterization of its genome (Tuskan et al. 2006) has eased the path for the study of AR. Trupiano et al. (2013) identified an AP2/ ERF TF (Pta ERF003) involved in stress 
responses and in adventitious and lateral root formation. The transcriptome analysis performed by Rigal et al. (2012) during AR in poplar enabled identification of several TFs from different families associated with different stages of AR. Among these TFs, Aintegumenta-Like 1 (PtAIL1) was induced during the early stages of AR and, together with PtAIL9 and the PtMYB36 of the MYB family, might form a regulatory network for AR formation in poplar. PtAIL-1, like PtaERF003, belongs to the Apetala2/Ethylene Responsive Factor family (AP2/ERF), and most TFs that exhibited changes in gene expression during the initial stages of AR in poplar have been included in this family (Rigal et al. 2012). The AP2/ERF is a plant-specific superfamily of TFs with 147 members in Arabidopsis and 200 in poplar (Zhuang et al. 2009). The ERF family, which is divided into two subfamilies and 12 groups, is the most extensive family of the 4 included in the AP2/ ERF superfamily (Ito et al. 2014).

The ERF-VII group of the AP2/ERF superfamily has been implicated in the response to several stress factors. ERF-VII genes carry a DNA-interaction domain (AP2) and a N-terminal degron motif (N-degron) that is linked to a turnover regulatory system (Papdi et al. 2015). The number of genes in this group varies from 3 in Vitis vinifera to 6 in Populus and 15 in Oryza sativa (Girardi et al. 2013). In Arabidopsis, the five members of the ERF-VII group are implicated in stress responses to submergence and low-oxygen conditions, showing their ability to activate or control glycolytic and fermentative pathways, leading to metabolic reprogramming (Licausi et al. 2010; Giuntoli et al. 2017; Eysholdt-Derszsó and Sauter 2019). The NDegron domain works as a destabilizing motif under normoxic conditions (Bailey-Serres et al. 2012). The responses to low-oxygen availability can be triggered by different types of stress, including wounding or flooding, which can induce ARs in several species (Steffens and Rasmussen 2016). Activity of the ERF-VII group therefore seems to be associated with the induction of new roots under specific conditions. Nonetheless, ERF-VIIs activity is not exclusively related to anaerobic responses, as chemical stimuli or pathogen-related stress can also induce their expression (Giuntoli et al. 2017).

In the present study, we analyzed the expression of an $A P 2 / E R F$ gene of the ERF-VII group of TFs in the early events of AR formation in in vitro microshoots of chestnut and oak. The gene, putatively named RAP2.12 like-1, was differentially expressed in juvenile (rootingcompetent) and mature (rooting-incompetent) microshoots during the induction of ARs, and the expression was also species-dependent. The effects of auxin, wounding and NPA on gene expression were evaluated in both species during the process. The findings provide some hints as to the relevance of the distinct factors involved in the induction of ARs in recalcitrant species of Fagaceae.

\section{Materials and methods}

\section{Plant materials and root induction}

Shoot culture lines previously established in vitro from basal shoots (BS) and crown branches (CR) of mature chestnut (Sanchez and Vieitez 1991) and oak (Vieitez et al. 1994) trees were used in this study. The chestnut lines were derived from an 80-year-old tree designated P2 (Sánchez and Vieitez 1991). The oak microshoots were established from a 300-year-old monumental tree named Saínza. The chestnut and oak lines are maintained in vitro as previously described by Ballester et al. (1999) and Vidal et al. (2003) respectively.

In chestnut, rooting experiments were performed by dipping the basal end of the shoot in a $4900 \mu \mathrm{M}$ aqueous solution of indole-3-butyric acid (IBA) for $1 \mathrm{~min}$. The dipped shoots were then transferred to $300 \mathrm{~mL}$ jars containing $50 \mathrm{ml}$ of basal rooting medium (BRM). The BRM 
consisted of Gresshoff and Doy (GD) medium (Gresshoff and Doy 1972) with 1/3-strength of macronutrients, supplemented with $30 \mathrm{~g} \mathrm{~L}^{-1}$ sucrose and $0.7 \%$ Bacto Difco agar. Shoots dipped in a mock solution were used as controls. To test the effect of NPA, auxin-treated shoots were incubated in BRM supplemented with $10 \mathrm{ml}$ of an aqueous filtered solution of $50 \mu \mathrm{M}$ NPA for 5 days, and the shoots were then transferred to BRM for 1 month. For qPCR analysis, shoots were harvested at the indicated sampling times (15 $\mathrm{min}, 6,12$ and $24 \mathrm{~h}$ ) after initiation of the treatments.

In oak, root induction was carried out by incubating the shoots for $24 \mathrm{~h}$ in root induction medium (RIM), which consisted of BRM supplemented with $125 \mu \mathrm{M}$ IBA. After auxin induction, shoots were transferred to BRM for 1 month. The NPA experiments were performed by adding $10 \mathrm{ml}$ of an aqueous filtered solution of $200 \mu \mathrm{M}$ NPA to the glass jars as a double phase. The NPA was first added to RIM during the $24 \mathrm{~h}$ auxin induction period, after which shoots were transferred to BRM supplemented with NPA. The effect of NPA on the rooting response was evaluated at the end of experiment (one month) in shoots treated with NPA for 5 days. For qPCR analysis, samples from control shoots, auxin treated shoots and shoots treated with IBA plus NPA were harvested at the end of the multiplication cycle (Time zero) as well as at $6,12,24,48$ and $72 \mathrm{~h}$ after initiation of the treatments.

\section{RNA extraction and qPCR analysis}

The analysis was performed with total RNA extracted from the basal parts $(1 \mathrm{~cm})$ of microshoots. At least fifteen shoots were pooled together to make each sample. Total RNA was extracted with the "Total RNA Purification Kit Plant/Fungi" (Norgen Biotek) following the manufacturer's instructions. RNA was treated with RQ1 RNase-free DNaseI (Promega) to eliminate residual DNA. Integrity of the RNA was analyzed by agarose gel electrophoresis, and the RNA concentration was measured in a Nanodrop spectrophotometer (Thermo Scientific).

cDNA synthesis and quantitative PCR were developed as previously described (Sánchez et al. 2007; Vielba et al. 2011). Briefly, $1 \mu \mathrm{g}$ of total RNA was reverse-transcribed using SuperScript II reverse transcriptase (Invitrogen), according to the manufacturer's instructions. The primers used for amplification of the selected amplicons were designed with Primer Express software version 3.0 (Applied Biosystems). RAP2.12 like-1 primers were designed according to the available sequences for the genes, accessible in the NCBI repository under the codes JF755969.1 (CsRAP2.12 like-1, chestnut) and KR152259.1 (QrRAP2.12 like-1, oak). The same set of genes, which amplify a fragment covering the end of the coding sequence and part of the 3'-UTR region, was used in both chestnut and oak experiments. The sequence of the Forward and Reverse primers were 5'- CATAGAGGCATTGTTCAGC-GG-3' and 5'TCTGGCCCATTCCAACCA-3' respectively. Three reference genes, i.e. Elongation Factor-1 (ELF-1), $\beta$-Tubulin (TUB) and Actin (ACT2), were used to normalize the expression of the target gene. These genes were selected due to their stability under the experimental conditions and were validated with geNORM software (Vandesompele et al. 2002). The genes have previously been used in our laboratory with satisfactory results (Corredoira et al. 2012). The following sequences of primers were used in their amplification: ELF-1 Forward (5'TGTGCCGTCCTCATTATTGACT-3'), ELF-1 Reverse: (5'-TCACGGGTCTGACCATCCTT3); TUB Forward (5'CTCGTGCTGTTCTCATGGATCT-3'); TUB Reverse (5'TGGCCGAAAACGAAGTTGTC-3'); ACT2 Forward (5'- GCCCCACGAGCTGTGTTC - $3^{\prime}$ ); and ACT2 Reverse (5'- TCTGGCCCATTCCAACCA -3'). Polymerase chain reactions were performed in an optical 48-well plate in a One-Step real-time PCR System (Applied Biosystems), and Power SYBR Green Master Mix was used to monitor double-stranded DNA synthesis. Reactions were carried out in a final volume of $15 \mu \mathrm{L}$, with $10.8 \mathrm{ng}$ of cDNA in each 
reaction. Three technical replicates for each treatment and time point were included in each PCR run. The relative expression value was calculated by the Comparative $\mathrm{Ct}$ Method (Schmittgen and Livak, 2008). DataAssist v3.0 software (Applied Biosystems) was used for all calculations and normalizations. The results are expressed as mean values ( \pm standard error) from at least three biological replicates. The data were then subjected to analysis of variance (ANOVA) followed by comparison of group means (Tukey-b test). Statistical analyses were performed with SPSS 25.0 (IBM).

\section{In situ hybridization}

In situ experiments were carried out as previously described (Vielba et al. 2011; Valladares et al. 2013). The basal part of BS and CR chestnut microshoots treated or not with IBA were collected after $12 \mathrm{~h}, 10$ and $14 \mathrm{~d}$ of culture in BRM. The apical parts of ARs that developed in IBA-treated BS microshoots were also harvested. Transverse and longitudinal cryosections were cut from samples embedded in Jung Tissue freezing medium and placed on 3-aminopropyltriethoxysilan-treated glass slides. A fragment of 200 bp of CsRAP2.12 like-1 in the nonconserved 3' region of cDNA was cloned into the PCR $®$ II vector (Invitrogen), which contained T7 and SP6 promoters and was used as a template for sense and antisense probes. Singlestranded, digoxigenin-labelled, gene-specific RNA probes were synthesized for CsRAP2.12 like-1 using the DIG RNA labelling kit (SP6/T7) as previously described (Aquea et al. 2009). The sense probe was used as a negative control. The hybridization signal was detected by incubation in the antidigoxigenin-alkaline phosphatase conjugate (Roche) and subsequent incubation in the chromogen NBT/BCIP (Sigma-Aldrich). Photographs were taken with an Olympus digital camera connected to a Nikon microscope under bright-field illumination.

\section{Results}

\section{Rooting experiments}

Chestnut microshoots of clone P2 treated with $4900 \mu \mathrm{M}$ IBA exhibited similar rooting percentages as previously described for the same treatments (Sanchez and Vieitez 1991; Vielba et al. 2020, this issue). High rooting percentages, ranging from 90 to $100 \%$, were achieved in BS microshoots after auxin treatment, whereas no roots were produced in CR microshoots. In BS shoots, the mean number of roots was 7.5, some of which (36\%) arose from the stem of the shoot (Fig. 1A-C). By contrast, the rooting capacity of BS shoots treated with IBA plus $50 \mu \mathrm{M}$ NPA decreased to $75 \%$ (data not shown). The number of roots was not affected by the presence of NPA; however, only $22 \%$ of ARs developed at the basal callus (Fig. 1B-D). In addition, there was a 5-day delay in the emergence of ARs in shoots treated with NPA, relative to IBA-treated shoots. The presence of NPA also had a negative effect on shoot appearance, as the shoots were less vigorous than those treated with IBA alone, and all displayed symptoms of vitrification, curly leaves and apical necrosis. Although NPA did not affect the mean length of the longest root, which ranged from 28.8 to $29.5 \mathrm{~mm}$, most of the roots that developed in the uppermost part of the shoots did not elongate under this treatment.

The rooting performance of oak shoots was similar to that previously described (Vidal et al. 2003). The rooting capacity of BS shoots of the Sainza clone was inhibited by $50 \%$ in the presence of NPA relative to that in shoots treated with IBA alone. 
qRT-PCR analysis of CsRAP2.12 like-1

Expression of CsRAP2.12 like-1 was analyzed in the basal sections of chestnut microshoots during the first $24 \mathrm{~h}$ after treatments (IBA, IBA+NPA and control). Figure 2A shows the pattern of expression of the CSRAP2.12 like-1 gene in BS microshoots. In all treatments, the levels of CsRAP2.12 like-1 expression were significantly higher in samples harvested after $6 \mathrm{~h}$ than in those harvested after $15 \mathrm{~min}$, but no differences were observed between treatments. Following this transient peak in response to wounding, expression of the gene decreased to initial levels. Although mRNA levels were not significantly affected by auxin or NPA treatments, expression levels were slightly higher in auxin treated shoots harvested after $12 \mathrm{~h}$ than in shoots not treated with auxin. Therefore, auxin seems to have a weak effect on gene expression after $12 \mathrm{~h}$, a conclusion supported by the fact that the presence of NPA inhibited the effect. Under the IBA+NPA treatment, the expression profile of CsRAP2.12 like-1 exhibited a similar trend to that observed in control shoots. These results showed a transient upregulation of CsRAP2.12 like-1 by wounding within the first $6 \mathrm{~h}$ of AR.

In mature microshoots (CR) a slight, but not statistically significant, increase in CsRAP2.12 like-1 mRNA levels was observed after $6 \mathrm{~h}$ in control samples, before decreasing to basal levels at $12 \mathrm{~h}$ (Fig. 2B). When the shoots were treated with auxin, the level of gene expression was similar to that of control shoots at each time point evaluated, although the relative mRNA values were higher in auxin-treated samples (not statistically significant; Fig. 2B). Interestingly, in control CR shoots, the mRNA levels were similar to those in BS shoots, whereas in auxintreated microshoots they were higher in mature than in juvenile-like shoots (Fig. 2B). Neither wounding nor auxin significantly altered expression of the gene (Fig. 2B). Samples treated with NPA were not analyzed here.

\section{In situ analysis}

To clarify the data described above and to characterize the expression profile of CsRAP2.12 like-1 at tissue level during the early steps of AR, we performed an in situ analysis of BS and CR microshoots treated or not treated with auxin. The analysis was carried out in samples harvested $12 \mathrm{~h}$ after treatment, because early cell divisions were observed in the cambial cells, within the first $24 \mathrm{~h}$ of the rooting process (Ballester et al. 1999). Furthermore, the qPCR revealed that auxin had a slight effect on gene expression at this time in BS shoots (Fig. 2). Samples collected 10, 14 and 30 days after auxin induction were also used for in situ mRNA localization. In the BS control microshoots collected $12 \mathrm{~h}$ after wounding, weak and diffuse expression of CsRAP2.12 like-1 was observed in the phloem (Fig. 3A), in accordance with the low expression levels detected by qPCR. By contrast, in auxin-treated juvenile shoots, transcripts were mainly observed in the cambium and derivative cells (Fig. 3B-C). Root primordia were developed in rooting competent shoots 10 and 14 days after auxin induction (Fig. 3D-E), whereas in rooting-incompetent shoots, cell division led to proliferation of phloem and cortex cells and callus formation (Fig. 3G-H).

Expression in non-rooting competent shoots was diffuse and very weak. High accumulation of CsRAP2.12 like-1 transcripts was observed in the root primordia (Fig. 3D-E). In the apex of ARs, the hybridization signal was detected in the cortical/endodermal initial cells and their derivatives, which give rise to the first cells of the endodermal and cortical files of the ground tissue (Fig. 3F). No specific signal was detected in sections hybridized with the sense probe (Fig. 3I) as the background signal was weaker in control sections than in sections hybridized with the antisense probe (Fig. 3I vs 3D). The in situ hybridization established a direct 
relationship between rooting competence and the location of CsRAP2.12 like-1 transcripts in cambial cells (regeneration-competent) that will become root founder cells. The location of the transcripts in the root meristem and the root apex suggests a possible role for the gene in controlling ground tissue patterning.

The in situ hybridization established a direct relationship between rooting competence and the location of CsRAP2.12 like-1 transcripts in cambial cells (regeneration-competent) that will become root founder cells. The location of the transcripts in the root meristem and the root apex suggests a possible role for the gene in controlling ground tissue patterning.

\section{qRT-PCR analysis of QrRAP2.12 like-1}

The expression of QrRAP2.12 like-1 was analyzed in both BS and CR oak microshoots during the first 72 hours of the root induction process (Fig. 4). The analysis was performed in control shoots, IBA-treated shoots and shoots treated with IBA plus NPA.

The ANOVA II (sampling time and treatment) applied to BS shoots data showed a significant effect of both factors, but no interaction between the two variables ( $p$ value 0.166). In BS microshoots, the lowest mRNA levels were generally detected in control samples, in which the expression level of $Q r R A P 2.12$ like-1 increased significantly at $6 \mathrm{~h}$ in response to basal cut (Fig. 4A). Increasing the sampling time from 6 to 12, 24, 48 and $72 \mathrm{~h}$ led to a significant decrease in the mRNA levels, but expression was maintained higher than at time zero. Therefore, wounding enhanced gene expression in BS microshoots. A similar gene expression pattern was observed in auxin treated shoots (Fig. 4A). Although auxin did not significantly alter the expression of QrRAP2.12 like-1, mRNA values in auxin-treated samples were higher than those in control shoots at $6 \mathrm{~h}$, suggesting a weak effect of the hormone in inducing the gene. The lack of a significant effect of auxin on gene expression may be due to the asynchronous initiation of root primordia. Interestingly, in shoots treated with IBA plus NPA, the expression profile was similar to that of IBA treated shoots, but expression levels were significantly higher than in control shoots (Fig. 4A). Hence, the expression of QrRAP2.12 like-1 was induced by wounding in juvenile-like microshoots. On the other hand, NPA appeared to promote expression of the QrRAP2.12 like-1 gene in auxin treated shoots.

During the time course analyzed, the levels of $Q r-R A P 2.12$ like-1 mRNA were always lower in CR microshoots than in BS shoots (Fig. 4A, B). In all treatments (control, IBA and IBA+NPA), expression of the gene was higher at $6 \mathrm{~h}$ than at time zero, after which there was a progressive and significant decline in mRNA levels, indicating a transient peak in expression in response to wounding. After $24 \mathrm{~h}$, the mRNA levels were similar to those at time zero, and after $72 \mathrm{~h}$, expression in IBA and IBA+NPA treated samples was lower than basal levels. At each sampling time, no differences between treatments were detected, indicating that the gene was not modulated by auxin. In non-rooting competent shoots, expression of the gene was therefore transiently upregulated by wounding, with a descending trend in the level of expression of the gene from $6 \mathrm{~h}$ onwards.

\section{Discussion}

Many factors influence the formation of ARs in cuttings of woody species. In this study, we characterized the expression profile of the RAP2.12 like-1 gene during the early steps of AR in chestnut and oak microshoots, and we explored the effect of NPA on the rooting response. In these species, both wounding and exogenous auxin are required to induce ARs in juvenile-like microshoots. However, in mature microshoots the same treatment leads to the formation of a callus structure, but no roots are generated. Maturation is associated with morphological and 
physiological changes during plant development and plays a crucial role in the plant responses to biotic and abiotic stimuli. Maturation-associated changes affect rooting capacity, hormone content, DNA methylation levels and wood density (Day et al. 2002; Wendling et al. 2014). The effects of phase change on AR and other traits in the culture lines used in this study have been characterized at anatomical, physiological, biochemical and molecular levels (Sánchez and Vieitez 1991; Vidal et al. 2003; Gil et al. 2004; Fernández-Lorenzo et al. 2005; Ballester et al. 2009). These changes are brought about by shifts in the expression patterns of transcription factors. At first glance, the different expression patterns of the RAP2.12 like-1 gene in BS and $\mathrm{CR}$ shoots of the same genotype but differing in their ability to form adventitious roots suggests the involvement of these genes in root regeneration.

CsRAP2.12 like-1 and QrRAP2.12 like-1 belong to group VII of the ERF transcription factor family (AP2/ ERF superfamily). In Arabidopsis, three out of five members of this group (AtRAP2.12, AtRAP2.2 and AtRAP2.3) act synergistically to integrate different stressors, including low oxygen, osmotic and oxidative stress, and improve the plant tolerance to these factors when they are overexpressed (Papdi et al. 2015). Their activity drives the metabolic reprogramming of the tissues, with a shift towards an anaerobic mode due to low oxygen availability, in order to prevent tissue damage (Giuntoli et al. 2017). Indeed, these are components of the oxygen and nitric oxide (NO) perception system, helping the plants mitigate the effects generated by these stressors. Furthermore, the stability of the resulting TFs is tightly regulated through different mechanisms in order to prevent their activity under normoxic conditions (Papdi et al. 2015). One of those mechanisms is the N-degron pathway, in which a conserved motif in the N-terminus of the protein acts as an oxygen-sensing mechanism that induces the degradation of the protein under normal oxygen conditions (Bailey-Serres et al. 2012; Eysholdt-Derszó and Sauter 2019). The presence of the N-degron motif in the putative protein encoded by oak and chestnut RAP2-12 like-1 genes suggests that these are also under specific control and may not be stable under normoxic conditions. Moreover, the role of ERFVII has been linked to development processes controlled through low-oxygen independent mechanisms involving ethylene, gibberellin and abscisic acid, as well as by interactions with other proteins (Giuntoli and Perata 2018). The activity of ERFs in some wounding processes is controlled via heterodimerization with other TFs, and this complex may induce cell division (Heyman et al. 2018).

The qPCR expression profile of CsRAP2.12 like-1 revealed that the gene was induced by wounding in BS shoots but not in CR shoots. Thus, the gene seems to be regulated by wounding in an ontogenetic-dependent manner. As already stated, only BS shoots are competent to form roots after the inductive treatment. There is therefore a direct relationship between rooting competence and wounding-induced expression of the gene. Changes in gene expression of members of the wound responsive genes were detected during the different stages of adventitious rooting in apple ( $\mathrm{Li}$ et al. 2018), but expression levels were affected by exogenous auxin. In our system, the wound-altered expression seems to be related to ethylene levels rather than to the exogenous IBA. Ethylene synthesis is promoted as a stress response to the basal cut performed to induce ARs and it has an important role in adventitious root formation (Harfouche 2008). A link between AR and altered expression levels of genes related to ethylene metabolism and signalling was observed during the AR of cuttings from Cryptomeria japonica D. Don (Fukuda et al. 2018). In oak, the analysis showed the upregulation of the gene in response to wounding in rooting competent shoots, which was particularly high at $6 \mathrm{~h}$, but the induction was maintained over the sampling time. By contrast, in CR shoots, the wounding effect was transient (at $6 \mathrm{~h}$ ) confirming that in both species the gene was altered in response to wounding in an ontogenetic-specific manner. Furthermore, the higher levels of RAP2.12 like-1 mRNA in oak 
than in chestnut control shoots and the different expression patterns after wounding indicate that wounding regulation of these genes is species-dependent.

The upregulation of RAP2.12 like-1 gene expression by wounding in BS shoots from chestnut and oak leads to a direct correlation between mRNA levels and the ability of shoots to form ARs, and suggests a role of the gene related to AR process. In petunia, transcriptomic analysis showed that 24 ERF genes were upregulated by wounding during AR induction (Druege et al. 2014). Interestingly, Heyman et al. (2018) reported that cell division induced by wounding was promoted by ERF113 (RAP2.6L), a gene responsive to ethylene with activity in shoot regeneration from roots, which also requires cell redifferentiation and acquisition of a new fate, similarly to de novo formation of ARs. It was recently reported that the expression of ERF RAP2.12 was upregulated during root induction from callus of Camellia sinensis (Gao et al. 2019).

qPCR analysis of CsRAP2.12 like-1 did not reveal any significant changes in expression of the gene after auxin treatment. These results may be related to the asynchronous responses of the shoots, or to the fact that particular responses to auxin are restricted to precise tissues or cells within the shoots. Collective analysis of all stem tissues may mask tissue-specific induction of the gene. In response to the root induction treatment, specific cells of BS shoots located within or close to the vascular bundles (cambial cells, cambial derivatives) are sensitive to the auxin signal, dedifferentiate and will eventually divide to initiate formation of the new structure (Ballester et al. 1999; Vidal et al. 2003). In many woody species, first cell divisions in response to auxin are observed in the cambium, from where callus and ARs are formed (Solé et al. 2008; de Almeida et al. 2015; Stevens et al. 2018). The observed trend in expression of CsRAP2.12 like-1 in CR shoots suggests that the gene may also be involved in the initial steps of the formation of the callus structure. Interestingly, the formation of callus is indeed a de novo organogenesis program that recapitulates some of the steps that lead to the formation of new roots (Sugimoto et al. 2011). Expression of AtERF114 was strongly induced after wounding, and overexpression promotes callus formation (Heyman et al. 2018).

The expression of CsRAP2.12 like-1 may therefore be related to the developmental programs induced by the different treatments, although tissue-resolution analysis is required to confirm this. To gain further insight into this issue, we conducted an in situ analysis with BS shoots to detect expression of this gene. We found that CsRAP2.12 like-1 is expressed in the tissues where the auxin response will trigger changes in the genetic developmental program that will initiate the new AR, mainly cambium and derivative cells. This specific location of the gene activity within the time-frame within which these cells are reorganizing but have not yet begun to divide (Ballester et al. 1999) suggests a putative role for the gene in AR formation in chestnut. Furthermore, expression of the gene in meristematic zones, root primordia and developing roots further indicates its putative relation with meristematic and cellular reprogramming activities. On the other hand, the weak expression in the callus developed in IBA treated CR shoots reinforces the role of the gene in the early stages of cell reprogramming rather than in cell proliferation. Thus, expression analysis in chestnut revealed that the gene is upregulated by wounding in a time and phase-dependent manner and by auxin in a cell typespecific manner.

A minor effect of auxin in oak BS shoots can be inferred from the different trends in expression observed after $6 \mathrm{~h}$ in BS and CR shoots. A trend toward increased expression after addition of IBA and IBA+NPA was found in BS shoots at $6 \mathrm{~h}$, whereas a downward trend in expression was observed in CR shoots. Moreover, in CR shoots, expression levels were less than one half the levels attained in BS shoots. As previously described in chestnut, regulation of 
QrRAP2.12-like 1 gene is ontogenetic-stage dependent. Based on the expression pattern in BS and CR shoots, the different regulation of the gene in both types of shoots, the relationship between lower expression in CR shoots and the lack of ability of mature shoots to form roots together suggest that the gene may be involved in the AR ability. QrRAP2.12 like-1 was therefore found to be influenced by wounding, auxin and the ontogenetic state in oak shoots.

The effect of the basal cut on the expression of wound-regulated genes appeared to be an essential early step towards the formation of ARs in cuttings (Da Costa et al. 2013). Nonetheless, in our experimental system a basal cut is necessary but not sufficient to induce ARs because exogenous auxin is essential for the generation of new roots. After wounding, endogenous auxin tends to accumulate at the base of the cutting as a consequence of PAT activity (Lup et al. 2016). When exogenous auxin is applied, movement of the hormone from both sources in BS microshoots appears to effectively create a gradient between neighboring tissues that will lead to the rooting response of specific cells. On the other hand, no such gradient is created in CR microshoots, and the auxin is evenly distributed within the tissues, triggering the formation of a callus structure, as monitored in chestnut by expression of the highly auxin-responsive gene CsGH3-1 (Vielba et al. 2016). This maturation related shift in auxin homeostasis has previously been suggested (Rasmussen et al. 2015).

Auxin induced the expression of CsRAP2.12-like-1 gene in rooting-competent shoots of chestnut (in situ analysis). The preferential accumulation of CsRAP2.12 like-1 transcripts in the cambial cells of IBA-treated BS shoots suggests a role for the gene in the early AR events. Although no direct relationship between ERF-VII TFs and AR has yet been established, some genes from this group were associated with the early stages of AR (Rigal et al. 2012; Wei et al. 2013; Wang et al. 2016). Furthermore, the specific location of Cs-RAP2.12 like-1 in the root apex indicates a putative function in controlling the cell division of the endodermis- and cortexderivative cells, preventing their differentiation and regulating the patterning of the root ground tissue. The activity of some ERF-VII members in Arabidopsis has been linked to root growth. Under hypoxic conditions, AtRAP2.12 influences auxin transport through its effect on the auxin transporter AtPIN-2 to prevent root slanting, while another member of the group, AtHRE2, controls the growth of the ARs induced by hypoxia (Eysholdt-Derszó and Sauter 2017, 2019). There is therefore a link between osmotic and oxidative stress, root formation and the activity of this group of genes. It has recently been shown that hypoxic niches are generated during the formation of lateral roots in Arabidopsis, which leads to the induction of ERF-VII genes, which in turn modulate the auxin response in the developing tissue (Shukla et al. 2019). Moreover, this hypoxic environment has also been detected in the shoot apical meristem, where oxygen acts as a diffusible signal that controls development (Weits et al. 2019). Thus, temporary hypoxic conditions may also influence the early stages of $\mathrm{AR}$, although further research is needed to clarify this issue.

The inhibitory effect of NPA on AR was clearly indicated in the root system of chestnut plantlets by the presence of a large number of small roots in the uppermost part of the stem. NPA apparently moved up the stem preventing endogenous auxin transport from the apex to the basal part of the shoot. The accumulation of endogenous auxin in the stem gives rise to the development of large numbers of roots outside the basal end of the shoot. Interestingly, in BS shoots, expression of QrRAP2.12 like-1 was higher in NPA treated samples than in control shoots. The fact that expression of QrRAP2.12 like-1 was not affected by exogenous auxin but was upregulated by NPA indicates a possible effect of endogenous auxin on expression of the gene. The higher AIA concentration in shoots treated with IBA plus NPA than in shoots treated with IBA alone, reported by Vidal et al. (2003) for the same working system, suggests a correlation between NPA, endogenous AIA content and expression of the Qr-RAP2.12 like-1 
gene in BS shoots. On the other hand, root emergence was delayed (2 days) in NPA-treated shoots (Vidal et al. 2003), and the peak expression detected after $72 \mathrm{~h}$ may therefore be related to a delay in the initial events (dedifferentiation or initial division of cells) that would lead to the development of AR primordia. Although different expression profiles were observed in chestnut and oak, species-dependent regulation of the gene by auxin cannot be confirmed, as oak and chestnut shoots were subjected to different concentrations of auxin and NPA as well as to different methods and periods of application of this hormone. Moreover, although qPCR analysis provided relevant information about the relative expression of genes, specific cell and tissue type distribution of transcripts are not easily detected by qPCR. In rooting competent cuttings of pine, auxin was detected in the cambial region whereas accumulation of auxin was not detected in similar cell types in non-competent hypocotyls or epicotyls (Abarca et al. 2014). Furthermore, these authors found that NPA treatment of rooting-competent hypocotyls resulted in the mislocation of endogenous auxin, which was also distributed throughout the cortex, pith and vascular cylinder (Abarca et al. 2014). In this respect, the use of NPA may be very helpful for unravelling the role of PAT in AR, a poorly characterized aspect (Da Costa et al. 2013). The targets of NPA within the auxin transport machinery have not been clearly established, because genetic and biochemical data indicate the involvement of different proteins (reviewed in Teale and Palme 2017). Nonetheless, specific interactions between NPA and the transport proteins may influence the outcome of the activity of this inhibitor. NPA can have different effects in closely related species (Saniewski et al. 2014). Our results in oak suggest that the effect of NPA is dependent on the ontogenetic stage of the shoots.

In the present work, we found that expression of the RAP2.12 like-1 genes was under ontogenetic control, suggesting that the processes to which they are related, such as stress responses and probably AR formation are differentially regulated in juvenile-like and adult plants in chestnut and oak. In a previous study, we reported the different expression profile of a member of the GRAS transcription factor family (CSSCL1) in juvenile-like and adult chestnut microshoots, with specific localization of transcripts in the cambial cells of rooting competent shoots (Vielba et al. 2011). In Arabidopsis, expression of ERF-VII genes is regulated in an agedependent manner (Giuntoli et al. 2017), suggesting that these genes may be involved in the different responses to stress during plant development. This is consistent with the differential expression of CsRAP2.12 like-1 and QrRAP2.12 like-1 to wounding depending on the ontogenetic stage of shoots. Furthermore, the pattern of expression of CsRAP2.12 like-1 at $12 \mathrm{~h}$ in BS microshoots (Fig. 3B) is very similar to that found for CsSCL-1 (Vielba et al. 2011). In Arabidopsis, ERF proteins (including ERF-VII) and GRAS proteins can heterodimerize, suggesting a cooperative mode of action of these TFs (reviewed in Heyman et al. 2018). It is therefore tempting to speculate that these two TFs interact in chestnut microshoots to govern specific gene expression. Future work may help confirm this hypothesis. Indeed, the involvement of GRAS and AP2/ ERF TFs in determining the fate of root founder cells in response to auxin during the AR process has previously been established (reviewed in Druege et al. 2016).

\section{Conclusions}

Expression analysis of CsRAP2.12 like-1 and QrRAP2.12 like-1 in juvenile-like and mature microshoots of Fagaceae species showed that the behaviour of the genes is affected by wounding in a species- and ontogenetic-dependent manner. Expression profiles of both genes suggests that they are under tight temporal control. The ontogenetic regulation of both genes indicates that they are involved in crosstalk between the maturation state of the tissues, stress conditions and hormone responses. Furthermore, the induction of AR is directly related to the presence of CsRAP2.12 like-1 transcripts in rooting competent cells. The putative role of 
RAP2.12 like-1 genes in AR and callus formation as well as in the control of root development suggest that these genes may have an important function in controlling the expression of downstream genes necessary for the correct establishment of new developmental programs.

Acknowledgments: This work was partially funded by the Xunta de Galicia through the Contrato Programa 2016-2018 and the project IN607A 2017/6.

\section{References}

Abarca, D., Pizarro, A., Hernández, I., Sánchez, C., Solana, S.P., del Amo, A., et al. (2014). The GRAS gene family in pine: transcript expression patterns associated with the maturation-related decline of competence to form adventitious roots. BMC Plant Biol. 14: 354. DOI: 10.1186/s12870-014-0354-8.

Aquea, F., Matte, J.P., Gutiérrez, F., Rico, S., Lamprecht, M., Sánchez, C., Arce-Johnson, P. (2009). Molecular characterization of a Trithorax-group homologue gene from Pinus radiata. Plant Cell Rep. 28: 1531-1538. DOI: 10.1007/s00299-009-0752-9.

Ballester, A., San-José, M.C., Vidal, N., Fernández-Lorenzo, J.L., Vieitez, A.M. (1999). Anatomical and biochemical events during in vitro rooting of microcuttings from juvenile and mature phases of chestnut. Ann. Bot. 83: 619-629. DOI: 10.1006/ anbo.1999.0865.

Ballester, A., Vidal, N., Vieitez, A.M. (2009). Developmental stages during in vitro rooting of hardwood trees from material with juvenile and mature characteristics. In: Adventitious root formation of forest trees and horticultural plants-from genes to applications. (Eds) K. Niemii, C. Scagel. Research Singpost, Kerala. 277-299.

Bailey-Serres, J., Fukao, T., Gibbs, D.J., Holdsworth, M.J., Lee, S.C., et al. (2012). Making sense of low oxygen sensing. Trends Plant Sci. 17: 129-38. DOI: 10.1016/j.tplants.2011.12.004.

Bonga, J.M. (1987). Clonal propagation of mature trees: problems and possible solutions. In Cell and Tissue Culture in Forestry. (Eds) J.M. Bonga and D.J. Durzan. Martinus Nijhoff Publishers, Dordrecht. 249-271. DOI: 10.1007/978-94-017-0994-1_15.

Corredoira, E., Valladares, S., Allona, I., Aragoncillo, C., Vieitez, A.M., Ballester, A. (2012). Genetic transformation of European chestnut somatic embryos with a native thaumatin-like protein (CsTL1) gene isolated from Castanea sativa seeds. Tree Physiol. 32:1389-1402. DOI: 10.1093/treephys/tps098.

Da Costa, C.T., de Almeida, M.R., Ruedell, C.M., Schwambach, J., Maraschin, F.S., Fett-Neto, A.G. (2013). When stress and development go hand in hand: main hormonal controls of adventitious rooting in cuttings. Front. Plant Sci. 4:133. DOI: 10.3389/fpls.2013.00133.

Day, M.E., Greenwood, M.S., Diaz-Sala, C. (2002). Age-and size-related trends in woody plant shoot development: regulatory pathways and evidence for genetic control. Tree Physiol. 22: 507-513. DOI: 10.1093/treephys/22.8.507.

De Almeida, M.R., Bastiani, D., Gaeta, M.L., Mariath, J.E.A., De Costa, F., Retallick, J., et al. (2015). Comparative transcriptional analysis provides insights into the molecular basis of adventitious rooting recalcitrance in Eucalyptus. Plant Sci. 239: 155-165. DOI: 10.1016/j.plantsci.2015.07.022.

Diaz-Sala, C. (2014). Direct reprogramming of adult somatic cells toward adventitious root formation in forest tree species: the effect of the juvenile-adult transition. Front. Plant Sci. 5: 310. DOI: 10.3389/fpls.2014.00310.

Druege, U., Franken, P., Hajirezaei, M.R. (2016). Plant hormone homeostasis, signaling and function during adventitious root formation in cuttings. Front. Plant Sci. 7: 381. DOI: 10.3389/fpls.2016.00381.

Druege, U., Franken, P., Lischewski, S., Ahkami, A. H., Zerche, S., Hause, B., Hajirezaei M.R. (2014). Transcriptomic analysis reveals ethylene as stimulator and auxin as regulator of adventitious root formation in petunia cuttings. Front. Plant. Sci. 5: 494. DOI: 10.3389/flps.2014.00494. 
Eysholdt-Derzsó, E., Sauter, M. (2017). Root bending is antagonistically affected by hypoxia and ERF-mediated transcription via auxin signaling. Plant Phys. 175: 412-423. DOI: 10.1104/pp.17.00555.

Eysholdt- Derzsó, E., Sauter, M. (2019). Hypoxia and the group VII ethylene response transcription factor HRE2 promote adventitious root elongation in Arabidopsis. Plant Biol. 21 (Suppl. 1): 103-108. DOI: 10.1111/plb.12873.

Fernández-Lorenzo, J., Ballester, A., Rigueiro, A. (2005). Phenolic content of microcuttings of adult chestnut along rooting induction. Plant Cell Tiss. Organ Cult. 83: 153-159. DOI: 10.1007/s11240005-4786-6.

Fukuda, Y., Hirao, T., Mishima, K., Ohira, M., Hiraoka, Y., Takahashi, M, Watanabe, A. (2018). Transcriptome dynamics of rooting zone and aboveground parts of cuttings during adventitious root formation in Cryptomeria japonica D. Don. BMC Plant Biology 18:201. DOI: 10.1186/s12870-0181401-7.

Gao, Y., Zhao, M., Wu, X., Li, D., Borthakur, D., Ye, J., Zheng, X., Lu, J. (2019). Analysis of differentially expressed genes in tissues of Camellia sinensis during dedifferentiation and root redifferentiation. Scientific Reports, 9: 2935. DOI: 10.1038/ s41598-019-39264-5.

Geiss, G., Gutierrez, L., Bellini, C. (2009). Adventitious root formation: new insights and perspectives. In: Annual Plant Reviews - Root Development. (Ed) Beeckman, T. Wiley-Blackwell. 37: 127-156. DOI: 10.1002/9781119312994.apr0400.

Gil, B., Pastoriza, E., Ballester, A., Sánchez, C. (2003). Isolation and characterization of a cDNA from Quercus robur differentially expressed in juvenile-like and mature shoots. Tree Physiol. 23: 633-640. DOI: 10.1093/treephys/23.9.633.

Girardi, C.L., Rombaldi, C.V., Dal Cero, J., Nobile, P.M., Laurens, F., Bouzayen, M., Quecini, V. (2013). Genome-wide analysis of the AP2/ERF superfamily in apple and transcriptional evidence of ERF involvement in scab pathogenesis. Sci. Hortic. 151: 112-121. DOI: 10.1016/j.scienta.2012.12.017.

Giuntoli, B., Perata, P. (2018). Group VII Ethylene response factors in Arabidopsis: Regulation and physiological roles. Plant Physiol. 176 (2): 1143-1155. DOI: 10.1104/pp.17.01225.

Giuntoli, B., Shukla, V., Maggiorelli, F., Giorgi, F.M., Lombardi, L., Perata, P., Licausi, F. (2017). Agedependent regulation of ERF-VII transcription factor activity in Arabidopsis thaliana. Plant Cell Environ. 40: 2333-2346. DOI: 10.1111/pce.13037.

Gresshoff, P.M., Doy, C.H. (1972). Development and differentiation of haploid Lycopersicon esculentum. Planta. 107: 161- 170. DOI: 10.1007/BF00387721.

Guan, L., Murphy, A., Peer, W., Gan, L., Li, Y., Chen, Z.M. (2015). Physiological and molecular regulation of adventitious root formation. Crit. Rev. Plant Sci. 34(5): 506-521. DOI: 10.1080/07352689.2015.1090831.

Hackett, W.P., Murray, J.R. (1996). Maturation or phase change. In: Molecular and morphological markers for juvenility, maturity, rejuvenation and somatic embryogenesis in woody plant species. (Eds) D. Thompson and M. Welander. European Commission, Malmo, Sweden, 7-22.

Harfouche, A., Rugini, E., Mencarelli, F., Botondi, R., Muleo, R. (2008). Salicylic acid induces H2O2 production and endochitinase gene expression but not ethylene biosynthesis in Castanea sativa in vitro model system. J Plant Physiol. 165(7):734-744. DOI: 10.1016/j.jplph.2007.03.010.

Heyman, J., Canher, B., Bisht, A., Christiaens, F., de Veylder, L. (2018). Emerging role of the plant ERF transcription factors in coordinating wound defense responses and repair. J Cell Sci. 131. DOI: $10.1242 /$ jcs. 208215 .

Ito, T.M., Polido, P.B., Rampim, M.C., Kaschuk, G., Souza, S.G. (2014). Genome-wide identification and phylogenetic analysis of the AP2/ERF gene superfamily in sweet orange (Citrus sinensis). Genet. Mol. Res. 3: 7839-7851. DOI: 10.4238/2014. September.26.22.

Lakehal, A., Bellini, C. (2019). Control of adventitious root formation: insights into synergistic and antagonistic hormonal interactions. Physiol. Plant. 165: 90-100. DOI: 10.1111/ ppl.12823. 
Legué, V., Rigal, A., Bhalerao, R.P. (2014). Adventitious root formation in tree species: involvement of transcription factors. Physiol. Plant. 151: 192-198. DOI: 10.1111/ppl.12197.

Li, K., Liang, Y., L. Xing, Mao, J., Liu, Z., Dong, F, Meng, Y., Han, M., Zhao, C, Bao, L., Zhang, D. (2018). Transcriptome analysis reveals multiple hormones, wounding and sugar signaling pathways mediate adventitious root formation in apple rootstock. Int. J. Mol. Sci. 19: 2201.DOI:10.3390/ijms 19082201.

Licausi, F., Dongen, J.T., Giuntoli, B., Novi, G., Santaniello, A., Geigenberger,- P., Perata, P. (2010). HRE1 and HRE2, two hypoxia- inducible ethylene response factors, affect anaerobic responses in Arabidopsis thaliana. Plant J. 62: 302-315. DOI: 10.1111/j.1365-313X.2010.04149.x.

Lup, S.D., Tian, X., Xu, J., Perez-Perez, J.M. (2016). Wound signaling of regenerative cell reprogramming. Plant Sci. 250: 178-187. DOI: 10.1016/j.plantsci.2016.06.012.

Papdi, C., Pérez-Salamó, I., Joseph, M., Giuntoli, B., Bögre, L., et al. (2015). The low oxygen, oxidative and osmotic stress responses synergistically act through the ethylene response factor VII genes RAP2.12, RAP2.2 and RAP2.3. Plant J. 82: 772- 784. DOI: 10.1111/tpj.12848.

Rasmussen, A., Hosseini, S.A., Hajirezaei, M.R., Druege, Geelen, D. (2015). Adventitious rooting declines with the vegetative to reproductive switch and involves a changed auxin homeostasis. J. Exp. Bot. 66:1437-1452. DOI: $10.1093 / \mathrm{jxb} /$ eru499.

Rigal, A., Yordanov, Y.S., Perrone, I., Karlberg, A., Tisserant, E., Bellini, C., et al. (2012). The AINTEGUMENTA LIKE1 homeotic transcription factor PtAIL1 controls the formation of adventitious root primordia in poplar. Plant Physiol. 160: 1996-2006. DOI: 10.1104/pp.112.204453.

Sánchez, M.C., Vieitez, A.M. (1991). In vitro morphogenetic competence of basal sprouts and crown branches of mature chestnut. Tree Physiol. 8: 59-70. DOI: 10.1093/treephys/8.1.59.

Sánchez, C., Vielba, J.M., Ferro, E., Covelo, G., Solé, A., Abarca, D., et al. (2007). Two SCARECROWLIKE genes are induced in response to exogenous auxin in rooting-competent cuttings of distantly related forest species. Tree Physiol. 27: 1459- 1470. DOI: 10.1093/treephys/27.10.1459.

Saniewski, M., Góraj, J., Węgrzynowicz-Lesiak, E., Miyamoto, K., Ueda, J. (2014). Differential effect of auxin transport inhibitors on rooting in some Crassulaceae species. Acta Agrobot. 67: 85-92. DOI: 10.5586/aa.2014.028.

Schmittgen, T.D., Livak, K.J. (2008). Analyzing real-Time PCR data by the comparative CT method. Nat. Protoc. 3: 1101-1108. DOI: 10.1038/nprot.2008.73.

Shukla, V., Lombardi, L., Iacopino, S., Pencik, A., Novak, O., Perata, P., Giuntoli, B., Licausi, F.(2019). Endogenous hypoxia in lateral root primordia controls root architecture by antagonizing auxin signaling in Arabidopsis. Mol. Plant. 12: 538-551. DOI: 10.1016/j.molp.2019.01.007.

Solé, A., Sánchez, C., Vielba, J.M., Valladares, S., Abarca, D., Díaz-Sala, C. (2008). Characterization and expression of a Pinus radiate putative ortholog to the Arabidopsis SHORT-ROOT gene. Tree Physiol. 28: 1629-1639. DOI: 10.1093/ treephys/28.11.1629.

Steffens, B., Rasmussen, A. (2016). The physiology of adventitious roots. Plant Physiol. 170: 603-617. DOI: $10.1104 /$ pp.15.01360.

Stevens, M.E., Woeste, K., Pijut, P.M. (2018). Localized gene expression changes during adventitious root formation in black walnut (Juglans nigra L.). Tree Physiol. 38: 877-894. DOI: 10.1093/treephys/tpx175.

Sugimoto, K., Gordon, S.P., Meyerowitz, E.M. (2011). Regeneration in plants and animals: dedifferentiation, transdifferentiation, or just differentiation? Trends Cell Biol. 21: 212-218. DOI: 10.1016/j.tcb.2010.12.004.

Teale, W., Palme, K. (2018). Naphthylphthalamic acid and the mechanism of polar auxin transport. $J$. Exp. Bot. 69: 303-312. DOI: 10.1093/jxb/erx323.

Trupiano, D., Yordanov, Y., Regan, S., Meilan, R., Tschaplinski, T., Scippa, G.S., Busov, V. (2013). Identification, characterization of an AP2/ERF transcription factor that promotes adventitious, lateral root formation in Populus. Planta 238: 271-282. DOI: 10.1007/s00425-013-1890-4. 
Tuskan, G.A., Difazio, S., Jansson, S., Bohlmann, J., et al. (2006). The genome of black cottonwood, Populus trichocarpa (Torr. and Gray). Science 313: 1596-1604. DOI: 10.1126/ science.1128691.

Valladares, S., Rico, S., Vieitez, A.M., Covelo, P., Sánchez, C. (2013). Expression of the QrCPE gene is associated with the induction and development of oak somatic embryos. Tree Genet. Genomes. 9: 1383-1393. DOI: 10.1007/s11295-013-0634-8.

Vandesompele, J., De Preter, K., Pattyn, F., Poppe, B., Van Roy, N., De Paepe, A., Speleman, F. (2002). Accurate normalization of real-time quantitative RT-PCR data by geometric averaging of multiple internal control genes. Genome Biol 3: (7) :re-search0034. DOI: 10.1186/gb-2002-3-7-research0034.

Vidal, N., Arellano, G., San-José, M.C., Vieitez, A., Ballester, A. (2003). Developmental stages during the rooting of in-vitro-cultured Quercus robur shoots from material of juvenile and mature origin. Tree Physiol. 23: 1247-1254. DOI: 10.1093/ treephys/23.18.1247.

Vieitez, A.M., Sánchez, M.C., J.B. Amo-Marco, Ballester, A. (1994). Forced flushing of branch segments as a method for obtaining reactive explants of mature Quercus robur trees for micropropagation. Plant Cell Tissue Organ Cult. 37:287-295. DOI: 10.1007/BF00042342.

Vielba, J.M., Díaz-Sala, C., Ferro, E., Rico, S., Lamprecht, M., Abarca, D., Ballester, A., Sánchez, C. (2011). CSSCL1 is differentially regulated upon maturation in chestnut microshoots and is specifically expressed in rooting-competent cells. Tree Physi-ol. 31(10): 1152-1160. DOI: 10.1093/treephys/tpr086.

Vielba, J.M., Varas, E., Rico, S., Covelo, P., Sánchez, C. (2016). Auxin-mediated expression of a GH3 gene in relation to ontogenic state in Chestnut. Trees - Struct. Funct. 30(6): 2237-2252. DOI: 10.1007/s00468-016-1449-7.

Wang, P., Ma, L.L., Li, Y., Wang, S.A., Li, L.F., Yang, R.T., Ma, Y.Z., Wang, Q. (2016). Transcriptome profiling of indole-3-butyric-acid induced adventitious root formation in softwood cuttings of the Catalpa bungei variety 'YU-1' at different developmental stages. Genes Genom. 38: 145-162. DOI: $10.1007 /$ s13258-015-0352-8.

Wei, K., Wang, L., Cheng, H., Zhang, C., Ma, C., Zhang, L., Gong, W., Wu, L. (2013). Identification of genes involved in indole-3-butyric acid induced adventitious root formation in nodal cuttings of Camellia sinensis (L.) by suppression subtractive hybridization. Gene 514: 91-98. DOI: 10.1016/j. gene.2012.11.008.

Weits, D.A., Kunkowska, A.B., Kamps, C.W., Portz, K.M. et al. (2019). An apical hypoxic niche sets the pace of shoot meristem activity. Nature 569: 714-717. DOI: 10.1038/s41586-019-1203-6.

Wendling, I., Trueman, S.J., Xavier, A. (2014). Maturation and related aspects in clonal forestry - Part I: concepts, regulation and consequences of phase change. New Forests 45: 449. DOI: 10.1007/s11056014-9421-0.

Zhuang, J., Peng, R.H., Cheng, Z.M., et al. (2009). Genome-wide analysis of the putative AP2/ERF family genes in Vitis vinifera. Sci Hortic-Amsterdam 123: 73-81. DOI: 10.1016/j. scienta.2009.08.002. 


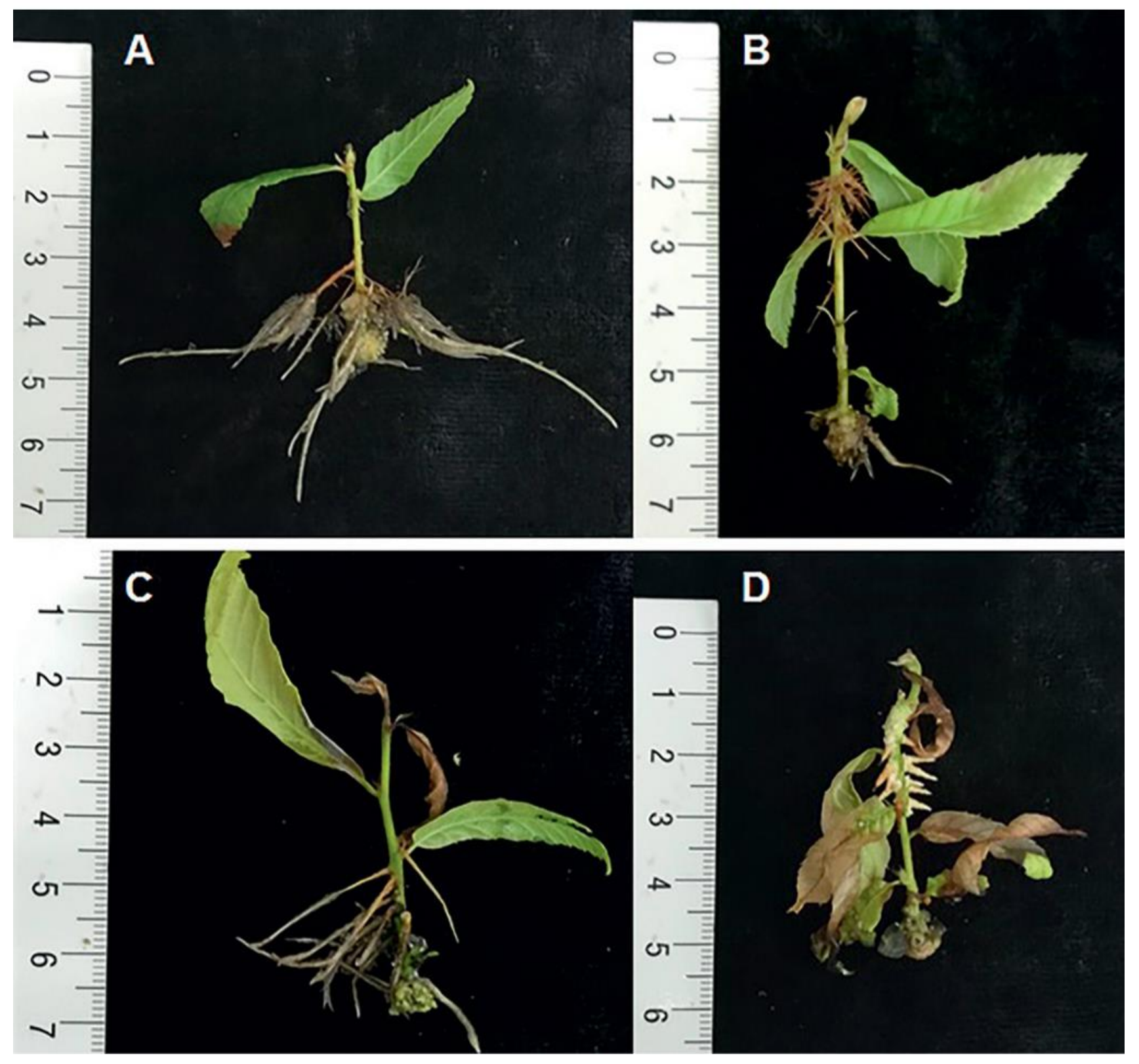

Figure 1. Phenotypes of chestnut plantlets rooted under different treatments. Microshoots were dipped in $4900 \mu \mathrm{M}$ IBA for 1 min and subsequently transferred to BRM for 1 month $(\mathrm{a}, \mathrm{c})$ or placed in BRM supplemented with an aqueous solution of $50 \mu \mathrm{M}$ NPA for 5 days and then transferred to BRM (b, d). 
A

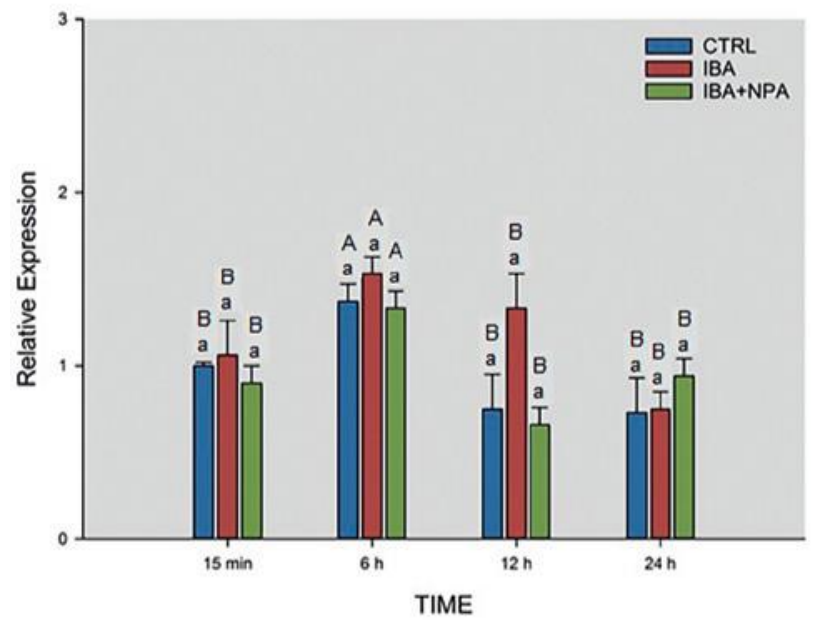

B

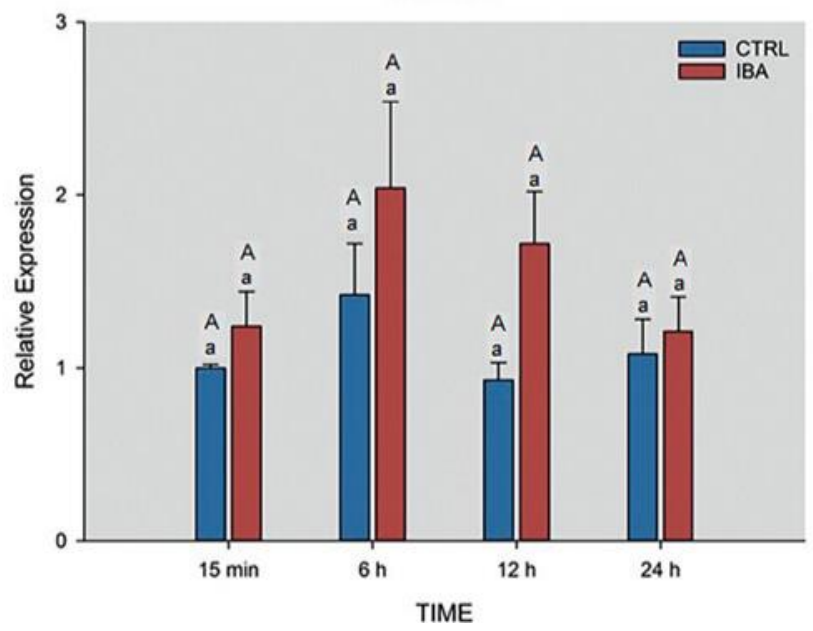

Figure 2. Expression of CsRAP2.12 like-1 gene in BS (A) and CR (B) chestnut microshoots. RNA was extracted from BS and CR control shoots (CTRL), from shoots treated with $4900 \mu \mathrm{M}$ IBA for 1 min (IBA), and from BS shoots treated with $4900 \mu \mathrm{M}$ IBA for 1 min and placed in BRM supplemented with an aqueous solution of $50 \mu \mathrm{M}$ NPA (IBA+NPA). qPCR was performed using total RNA extracted from the basal parts of shoots subjected to the different treatments and harvested at the indicated times. Results are expressed as mean values of relative expression $\pm \mathrm{SE}$ from three biological replicates. All data were normalized to time $15 \mathrm{~min}$. For each shoot type, different capital letters indicate significant differences in relation to the sampling time and different small letters indicate significant differences in relation to the treatments $(\mathrm{p} \leq 0.05)$.

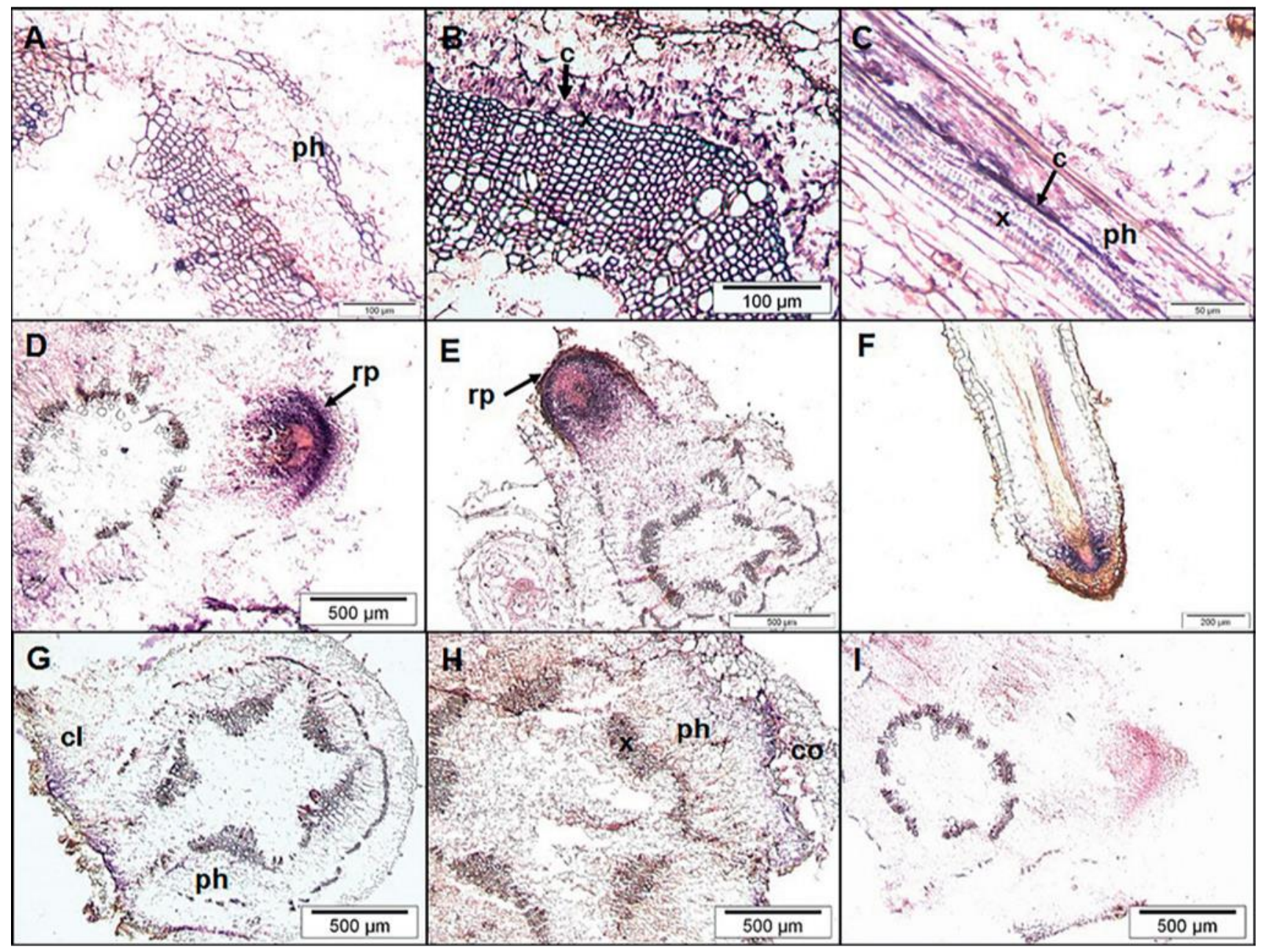


Figure 3(previous page). In situ localization of CsRAP2.12 like-1 transcripts in transverse sections of chestnut microshoots treated with IBA (B, D, E, G, H, J) or not treated (A), and in longitudinal sections from IBA-treated BS shoots $(C)$ and from AR developed in auxin-treated BS shoots (F). A weak signal was detected in untreated BS microshoots (A), and in IBA-treated CR samples (G, H) cultured for $12 \mathrm{~h}(\mathrm{~A}), 10(\mathrm{G})$ or 14 days $(\mathrm{H})$ in BRM. Specific accumulation of transcripts was detected in the cambial cells (c) of IBA-treated shoots harvested after $12 \mathrm{~h}$ (B, C). A strong signal was observed in the root primordia (rp) developed in BS microshoots after 10 (D) or 14 days of IBA treatment. No signal was detected in the root primordia hybridized with the sense probe (I). c, cambium; cl, calli; ph, phloem; rp, root primordia.

A

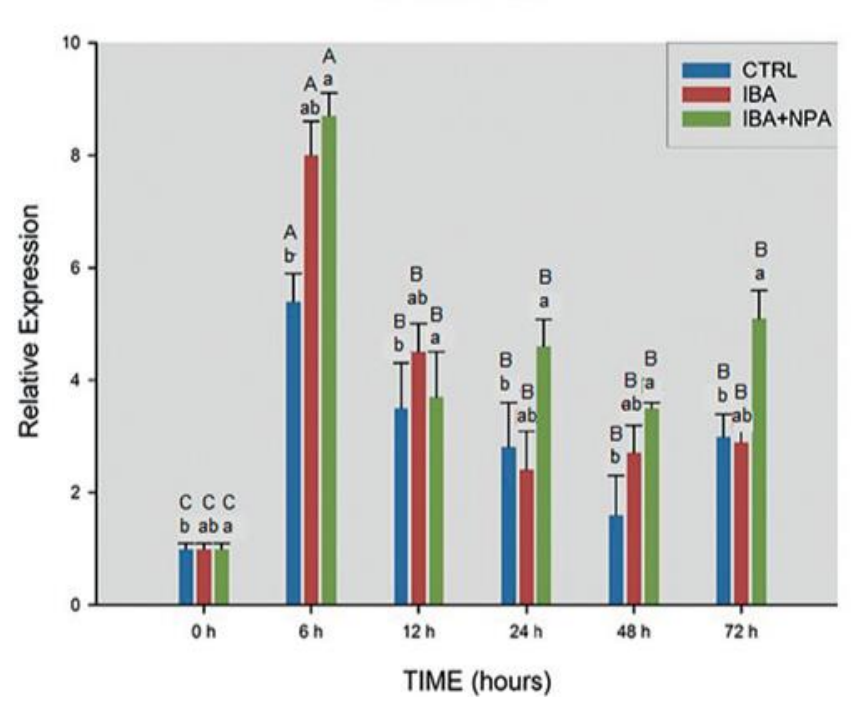

B

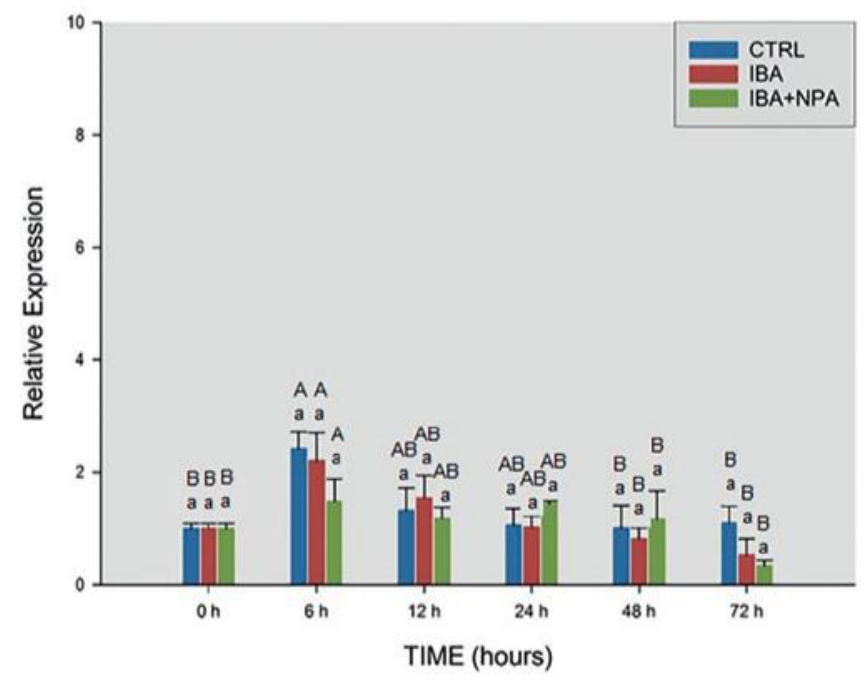

Figure 4. Expression of QrRAP2.12 like-1 gene in BS (A) and CR (B) microshoots of oak. RNA was extracted from control shoots (CTRL), from shoots treated with $125 \mu \mathrm{M}$ IBA for $24 \mathrm{~h}$ (IBA), and from shoots treated with $125 \mu \mathrm{M}$ IBA plus an aqueous solution of $200 \mu \mathrm{M}$ NPA for $24 \mathrm{~h}$ and subsequently transferred to BRM supplemented with an aqueous solution of $200 \mu \mathrm{M}$ NPA. qPCR was performed using total RNA extracted from the basal part of shoots subjected to the different treatments and harvested at the indicated times. Results are expressed as mean values of relative expression $\pm \mathrm{SE}$ from three biological replicates. All data were normalized to time 0 . For each shoot type, different capital letters indicate significant differences in relation to the sampling time and different small letters indicate significant differences in relation to the treatments $(\mathrm{p} \leq 0.05)$. 